\title{
Программная модель для исследования эффективности прочедур выделения контуров зашумленных изображений
}

\author{
E.A. Самойлин 1, д.т.н., профрессор, es977@mail.ru \\ C.A. Карпов ${ }^{1}$, ад'ъюнкт, sergei-andreevich@bk.ru
}

1 Военный учебно-научный иентр Военно-воздушных сил "Военно-воздушная академия uм. проф. Н.Е. Жуковского и Ю.А. Гагарина", г. Воронеж, 394064, Россия

В статье развивается одно из направлений цифровой обработки изображений - задача выделения их контуров.

Данная задача решается в интересах последующего распознавания объектов на изображении (по форме границ, углам между фрагментами контуров и т.п.), улучшения визуального качества изображения, подчеркивания границ объектов интереса, контрастирования и т.д. Цифровые изображения на практике регистрируются, как правило, в сложных и неблагоприятных условиях, что приводит к возникновению на них помех, описывающихся аддитивной гауссовской моделью шума. В соответствии с этим предложена программная модель, позволяющая исследовать эффективность самых различных методов, алгоритмов и процедур выделения контуров зашумленных изображений.

Модель основана на сопоставлении контура изображения, полученного из зашумленного изображения, с идеальным контуром, полученным из аналогичного изображения без помех, и на последующем вычислении ошибок обнаружения контурных элементов первого (ложная тревога) и второго (пропуск) рода. При этом вычисление указанных ошибок осуществляется во всем возможном диапазоне среднеквадратического отклонения $\sigma$ аддитивного гауссовского шума.

Представлены примеры выделения контуров зашумленных изображений реальных сцен, а также зависимости частот ошибок первого и второго рода от среднеквадратического отклонения шума амплитуды $\sigma$ от 0 до 200 (изображения при 256 уровнях квантования амплитуды яркости) для трех процедур выделения контура: на основе масок Превитта, Собела и лапласиана гауссиана. Кроме того, исследовано влияние процедуры линейной фильтрации зашумленного изображения на снижение частоты ошибок выделения контуров первого и второго рода.

Ключевые слова: изображение, выделение контуров, аддитивный гауссовский иум, градиентные маски, маски Превитта, маски Собела, лапласиан гауссиана.

Сегодня практически во всех отраслях науки и техники нет ни одной области, которая не нуждалась бы в визуальном представлении информации. Визуальная информация (фото, видеофайлы, голограммы и многое другое) хранится и воспроизводится в цифровом виде. Поэтому в различных сферах медицины, астрономии, микроскопии, лазерной техники, промышленности, правоохранительной деятельности широко применяют разнообразные компоненты для цифровой обработки изображений [1]. Существует множество программных продуктов и БД, таких как MATLAB, MATHCAD, OpenCV и т.д., которые в своем составе имеют самостоятельные модули, позволяющие обрабатывать цифровые изображения.

Цифровая обработка изображений нацелена на повышение визуального качества (использование различных фильтров) и выделение необходимых для пользователя информативных элементов обрабатываемого изображения (например, выделение контуров, ключевых точек и т.д.) [1].

Важным информативным элементом изображения является его контурный состав [2-4], который может использоваться для последующего распознавания объектов, выделения областей интереса и т.д. Существующие процедуры выделения контуров изображений [1-7] построены на обнаружении резких локальных перепадов яркости. Для обнаружения таких перепадов особенно хорошо подходят производные 1-го и 2-го порядков $[1,5,7]$. Выделение контура с использованием производной 1-го порядка обычно предполагает нахождение градиента по операторам Превитта или Собела и дальнейшую пороговую обработку [1]. Детекторы контуров Марра-Хилдрета и Кэнни (нахождение 2-й производной) построены на использовании выражения лапласиана гауссиана [1].

На практике съемка нередко происходит в различных неблагоприятных условиях: туман и повышенная влажность, предельно низкая освещенность, критические температуры, механические вибрации цифрового фотоприемника $[1,5,7]$. Это приводит к появлению на регистрируемом изображении помех, которые чаще всего могут описываться моделью аддитивного гауссовского шума (АГШ) $[1,5]$. При наличии на изображении АГШ выделение (обнаружение) контурных элементов процедурами на основе как 1-й, так и 2-й производных будет осуществляться с ошибками. Поэтому в настоящее время актуальна задача исследования эффективности процедур выделения контуров изображений, искаженных АГШ.

Целью данной работы является разработка программной модели, позволяющей исследовать эффективность различных процедур выделения контуров зашумленных изображений.

\section{Математические модели изображений и процедур выделения контуров}

Математическая модель неискаженного цифрового изображения представляется в виде матрицы 
$\boldsymbol{\Lambda}$ с матричными элементами $\lambda_{i, j}(i, j-$ номер строки и столбца соответственно), численные значения которых показывают амплитуду яркости в соответствующих точках изображения [1]:

$$
\lambda_{i, j} \in\left[1 \ldots 2^{N}\right], N=8,
$$

где $N$ - степень квантования амплитуды яркости; $2^{N}$ - число уровней квантования $\lambda_{i, j}$.

Искажение исходного изображения (1) АГШ предполагает изменение значений яркости каждого элемента изображения (1) на случайную величину с нулевым матожиданием и некоторым значением среднеквадратического отклонения (СКО). В соответствии с этим элементы изображения (1), полученного в условиях АГШ, могут быть представлены матрицей $\mathbf{X}$ с матричными элементами:

$$
x_{i, j .}=\lambda_{i, j} .+h_{i, j} \text {, }
$$

где $h_{i, j}-$ случайная величина с нулевым матожиданием и СКО $\sigma[1]$.

На практике значения СКО могут быть различными, от небольших (высококачественное изображение) до высоких (сильно разрушенное изображение). В качестве примера на рисунке 1 показаны исходное изображение $\boldsymbol{\Lambda}$ (1) и соответствующие ему зашумленные изображения $\mathbf{X}$ (2) с величиной СКО от 50 до 250 уровней амплитуды (при 256 квантованных уровнях амплитуды, то есть $N=8$ ).

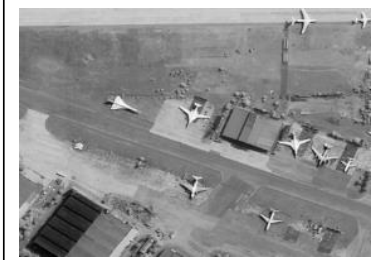

a)

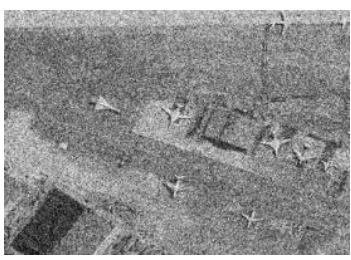

в)

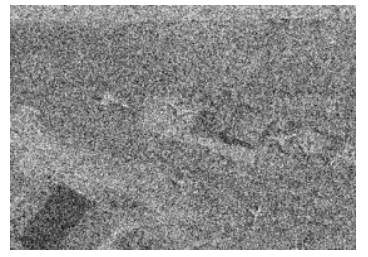

d)

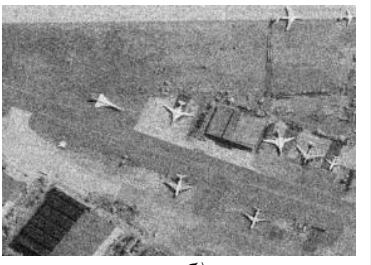

б)

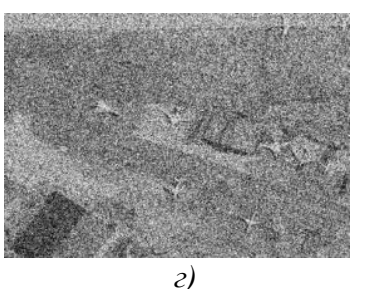

2)

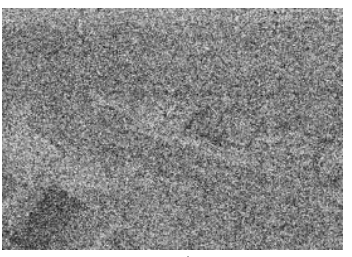

e)
Рис. 1. Влияние величины СКО на результат визуального искажения изображения: а) исходное изображение, б) зашумление при $\sigma=50$, в) зашумление при $\sigma=100$, г) зашумление при $\sigma=150$, д) зашумление при $\sigma=200$, е) зашумление при $\sigma=250$

Fig. 1. The effect of the mean square deviation value on the visual image distortion result: a) source image, б) noise at $\sigma=50$, в) noise at $\sigma=100$, г) noise at $\sigma=150$, d) noise at $\sigma=200$, e) noise at $\sigma=250$
Очевидно, что при $\sigma>200$ изображение практически не воспринимается, поэтому выделение контура объекта интереса на таком изображении невозможно.

Для получения контуров изображения $\mathbf{X}$ (2) с использованием производных 1-го порядка известно [1-4] выражение

$$
k_{i, j}^{\mathbf{X}}=\left\{\begin{array}{l}
1 \text { при } \nabla_{i, j} \geq P, \\
0 \text { при } \nabla_{i, j}<P,
\end{array}\right.
$$

где $k_{i, j}^{\mathbf{X}}$ - элемент матрицы $\mathbf{K}^{\mathbf{x}}$, полученной из изображения $\mathbf{X}(2) ; P$ - величина порога обнаружения; $\nabla_{i, j}-$ градиент изображения $\mathbf{X}(2)$ в точке $i, j$, определяемый по формуле

$\nabla_{i, j}=\left(\left(\sum_{s=-1}^{1} \sum_{q=-1}^{1} x_{i+s, j+q} g 1_{s, q}\right)^{2}+\left(\sum_{s=-1}^{1} \sum_{q=-1}^{1} x_{i+s, j+q} g 2_{s, q}\right)^{2}+\ldots\right.$

$\left.\ldots+\left(\sum_{s=-1}^{1} \sum_{q=-1}^{1} x_{i+s, j+q} g 3_{s, q}\right)^{2}+\left(\sum_{s=-1}^{1} \sum_{q=-1}^{1} x_{i+s, j+q} g 4_{s, q}\right)^{2}\right)^{\frac{1}{2}}$,

где $g 1_{s, q}, g 2_{s, q}, g 3_{s, q}, g 4_{s, q}$ - элементы разноориентированных градиентных масок для выделения составляющих градиента поля изображения вдоль столбцов $\left(g 1_{s, q}\right)$, строк $\left(g 2_{s, q}\right)$, главной $\left(g 3_{s, q}\right)$ и второстепенной $\left(g 4_{s, q}\right)$ диагоналей соответственно; $s, q=(-1,0,1)$ - индексы элементов масок, определяющие ее размер ( $3 \times 3$ в данном случае) [1].

При этом маски с элементами $g 1_{s, q}, g 2_{s, q}, g 3_{s, q}, g 4_{s, q}$ для оператора Превитта [1] имеют следующий вид:

$$
\begin{aligned}
& \mathbf{G 1}=\left[\begin{array}{ccc}
g 1_{-1,-1} & g 1_{-1,0} & g 1_{-1,1} \\
g 1_{0,-1} & g 1_{0,0} & g 1_{0,1} \\
g 1_{1,-1} & g 1_{1,0} & g 1_{1,1}
\end{array}\right]=\left[\begin{array}{ccc}
-1 & -1 & -1 \\
0 & 0 & 0 \\
1 & 1 & 1
\end{array}\right], \\
& \mathbf{G 2}=\left[\begin{array}{ccc}
-1 & 0 & 1 \\
-1 & 0 & 1 \\
-1 & 0 & 1
\end{array}\right], \mathbf{G 3}=\left[\begin{array}{ccc}
-1 & -1 & 0 \\
-1 & 0 & 1 \\
0 & 1 & 1
\end{array}\right], \\
& \mathbf{G 4}=\left[\begin{array}{ccc}
0 & 1 & 1 \\
-1 & 0 & 1 \\
-1 & -1 & 0
\end{array}\right] .
\end{aligned}
$$

Аналогичные маски для оператора Собела [1] имеют схожий вид:

$$
\begin{aligned}
& \mathbf{G 1}=\left[\begin{array}{ccc}
-1 & -2 & -1 \\
0 & 0 & 0 \\
1 & 2 & 1
\end{array}\right], \mathbf{G 2}=\left[\begin{array}{ccc}
-1 & 0 & 1 \\
-2 & 0 & 2 \\
-1 & 0 & 1
\end{array}\right], \\
& \mathbf{G 3}=\left[\begin{array}{ccc}
-2 & -1 & 0 \\
-1 & 0 & 1 \\
0 & 1 & 2
\end{array}\right], \mathbf{G 4}=\left[\begin{array}{ccc}
0 & 1 & 2 \\
-1 & 0 & 1 \\
-2 & -1 & 0
\end{array}\right] .
\end{aligned}
$$

Для получения контуров изображения $\mathbf{X}$ (2) с использованием производной 2-го порядка можно воспользоваться выражением (3), где вместо градиента $\nabla$ используется оператор лапласиана гауссиана $F[1,3]$, определяемый по формуле

$$
F_{i, j}=\left|\sum_{s=-2}^{2} \sum_{q=-2}^{2} x_{i+s, j+q} g_{s, q}\right|,
$$


где $g_{s, q}$ - элементы маски для выделения перепадов яркости изображения; $s, q=(-2,-1,0,1,2)-$ индексы элементов маски, определяющие ее размер ( $5 \times 5$ в данном случае).

Маска $\mathbf{G}$ с элементами $g_{s, q}[1]$ имеет следующий вид:

$$
\begin{aligned}
& \mathbf{G}=\left[\begin{array}{lllll}
g_{-2,-2} & g_{-2,-1} & g_{-2,0} & g_{-2,1} & g_{-2,2} \\
g_{-1,-2} & g_{-1,-1} & g_{-1,0} & g_{-1,1} & g_{-1,2} \\
g_{0,-2} & g_{0,-1} & g_{0,0} & g_{0,1} & g_{0,2} \\
g_{1,-2} & g_{1,-1} & g_{1,0} & g_{1,1} & g_{1,2} \\
g_{2,-2} & g_{2,-1} & g_{2,0} & g_{2,1} & g_{2,2}
\end{array}\right]= \\
& =\left[\begin{array}{ccccc}
0 & 0 & -1 & 0 & 0 \\
0 & -1 & -2 & -1 & 0 \\
-1 & -2 & 16 & -2 & -1 \\
0 & -1 & -2 & -1 & 0 \\
0 & 0 & -1 & 0 & 0
\end{array}\right] .
\end{aligned}
$$

\section{Модель исследования эффективности процедур выделения контуров}

Предлагаемая модель исследования эффективности процедур выделения контуров цифровых изображений в условиях АГШ выглядит следующим образом [8].

Допустим, необходимо сопоставить по эффективности три процедуры выделения контура: на основе масок Превитта (5), Собела (6) и лапласиана гауссиана (8). Для этого на первом этапе путем обработки тестового незашумленного изображения $\boldsymbol{\Lambda}$ (например, показанного на рисунке 1а) осуществляется построение трех вариантов его контуров: на основе масок Превитта (5), Собела (6) и лапласиана гауссиана (8). Полученные таким образом три варианта контуров $\mathbf{K}^{\mathbf{\Lambda}}$ изображения $\boldsymbol{\Lambda}$ назовем идеальными контурами для операторов Превитта (5), Собела (6) и лапласиана гауссиана (8) соответственно. Примеры таких идеальных контуров, построенных из изображения рис. 1а тремя разными процедурами, показаны на рисунке 2.

На втором этапе осуществляется зашумление тестового изображения $\boldsymbol{\Lambda}$ (например, показанного на рисунке 1а) АГШ с небольшим значением СКО $\sigma(2)$ [8]. Далее путем обработки этого зашумленного изображения $\mathbf{X}$ (2) осуществляется построение аналогичных трех вариантов его контуров $\mathbf{K}^{\mathbf{X}}$ : на основе масок Превитта (5), Собела (6) и лапласиана гауссиана (8). На рисунке 3 представлены результаты такого выделения контуров из изображения (рис. 1a), зашумленного АГШ с $\sigma=20$.

Рисунок показывает, что при наличии на изображении шума определение контурных элементов осуществляется с ошибками. Как известно из статистической радиотехники [9], возможны четыре исхода при обнаружении сигнала (то есть контурного элемента) на изображении $\mathbf{X}$ : две ситуации, связанные с его правильным обнаружением либо

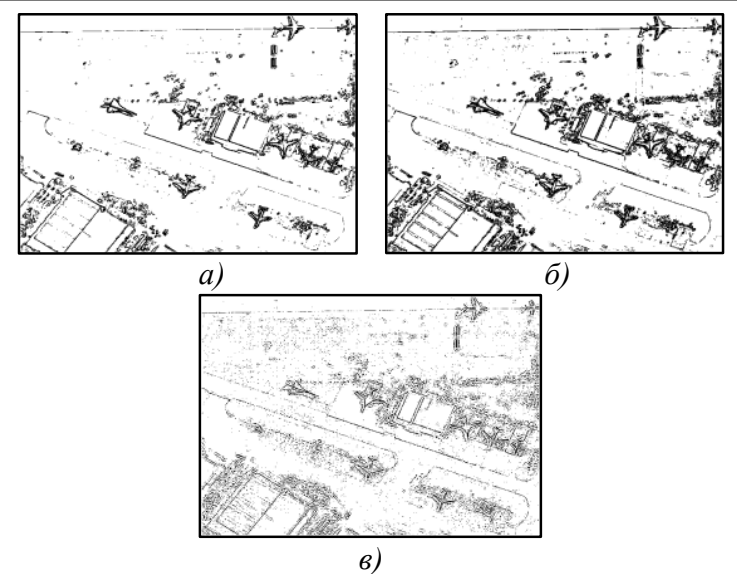

Рис. 2. Получение идеального контура из исходного изображения (показанного на рисунке 1а): а) маски Превитта (5), б) маски Собела (6), в) лапласиан гауссиана (8)

Fig. 2. Getting the ideal contour from the source image (shown in Fig. 1a): a) Prewitt masks (5),

б) Sobel masks (6), в) Laplacian of Gaussian (8)

необнаружением, и две ошибочные ситуации, связанные с ошибками типа «ложная тревога» (ошибка 1-го рода) и «пропуск» (ошибка 2-го рода). Поэтому на третьем этапе для расчета ошибок 1-го и 2-го рода необходимо полученные с использованием масок Превитта, Собела и лапласиана гауссиана контуры (рис. 3) $\mathbf{K}^{\mathbf{x}}$ попиксельно сопоставить с полученными аналогичными масками идеальными контурами (рис. 2) $\mathbf{K}^{\Lambda}$. Для расчета величин этих ошибок необходимо для всех трех пар изображений вычислить разности (найти матрицу С) между контурными матрицами $\mathbf{K}^{\boldsymbol{\Lambda}}$ и $\mathbf{K}^{\mathbf{X}}$.

$$
c_{i, j}=k_{i, j}^{\lambda}-k_{i, j}^{x},
$$

где $k_{i, j}^{\lambda}$ и $k_{i, j}^{x}$ - матричные элементы соответ-

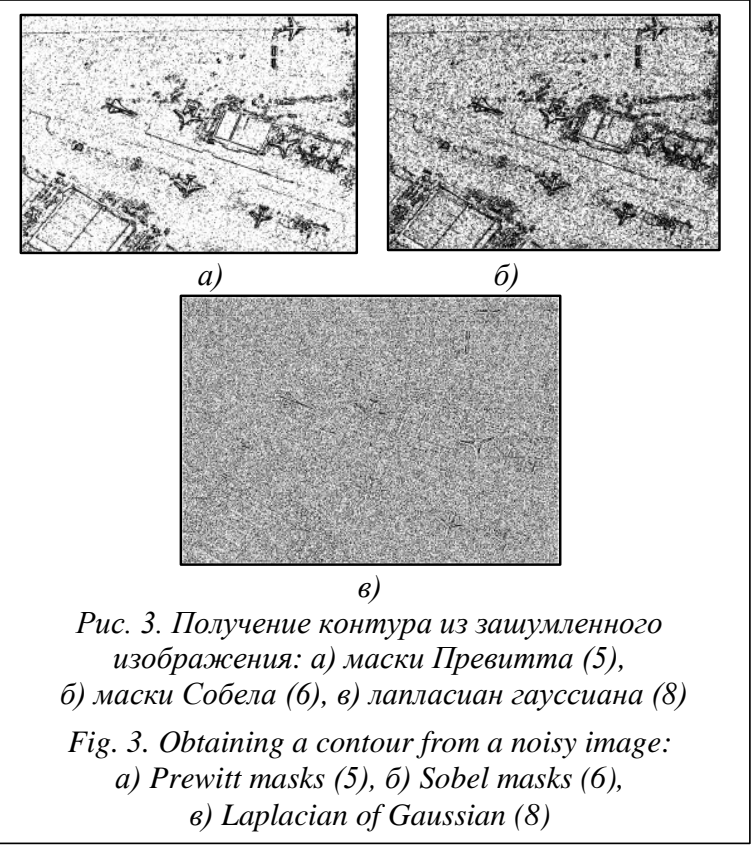




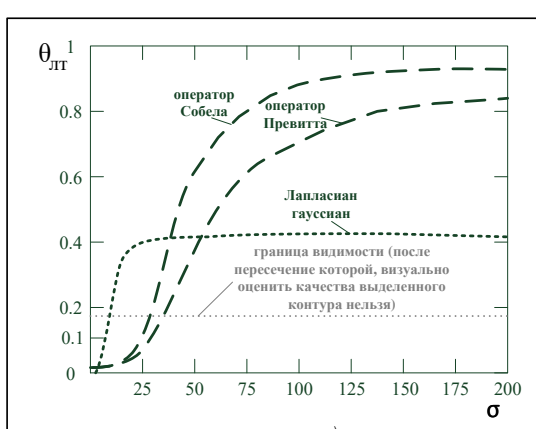

a)

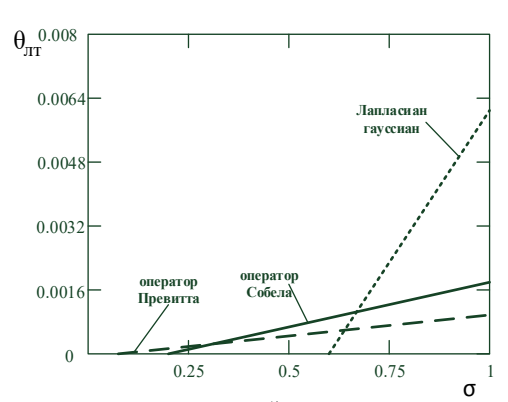

б)

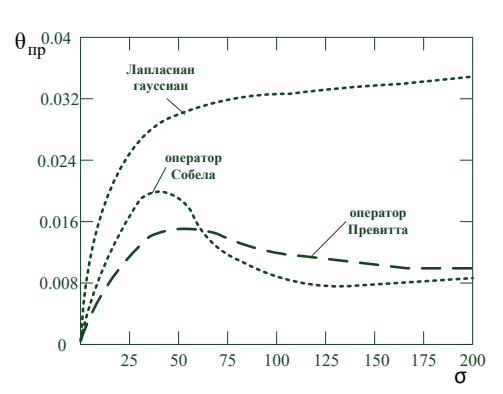

в)

Рис. 4. Ошибки сопоставляемых масочных операторов выделения контура: а) ошибки 1-го рода (во всем диапазоне б), б) ошибки 1-го рода (при $\sigma<1)$, в) ошибки 2-го рода (во всем диапазоне б)

Fig. 4. Errors of matched mapped contour selection operators: a) errors of the 1st kind (in full range of $\sigma$ ), б) errors of the 1st kind (when $\sigma<1$ ), 6 ) errors of the 2 nd kind (in full range of $\sigma$ )

ственно идеального контура $\mathbf{K}^{\mathbf{\Lambda}}$ и контура $\mathbf{K}^{\mathbf{X}}$ изображения, полученного при наличии на нем помех.

Затем для разделения ошибок 1-го и 2-го рода можно воспользоваться формулами подсчета частот событий, заключающихся в ошибках типа «ложная тревога» $\left(\theta_{\text {лг }}\right)$ и «пропуск» $\left(\theta_{\text {пр. }}\right)$ контура соответственно:

$$
\theta_{\text {лт }}=\frac{1}{I J} \sum_{i=1}^{I} \sum_{j=1}^{J} c_{i, j} \text { при } c_{i, j}<0,
$$

где $I$ - количество матричных элементов в строке; $J$ - их число в столбце,

$$
\theta_{\text {пр. }}=\frac{1}{I J} \sum_{i=1}^{I} \sum_{j=1}^{J} c_{i, j} \text { при } c_{i, j}>0 \text {. }
$$

На рисунке 4 представлены зависимости частот (вероятностей) ошибок 1-го и 2-го рода от СКО, построенные в соответствии с предлагаемой программной моделью [8]. В рассматриваемом примере для операторов на основе масок Превитта и Собела величина порога обнаружения $P$ (3) задавалась равной 150. Для оператора лапласиана гауссиана уровень пересечения нулевого значения принимался равным 100.

Из рисунка 4 видно, что в области низких значений СКО $(\sigma<0,6)$ более высоким качеством выделения контура обладает лапласиан гауссиана. По мере роста СКО $(0,6<\sigma<35)$ последний показывает большие значения ошибок 1-го и 2-го рода по сравнению с градиентными операторами на основе 1-й производной (масками Превитта и Собела), что соответствует выводам $[1,3,5]$ о более высокой помехоустойчивости последних по сравнению с операторами на основе 2-й производной. Сравнивая между собой операторы на основе масок Превитта и Собела, можно отметить более высокую устойчивость к помехам первых в отношении ошибок 1-го рода (рис. 4a, 4б), а также более высокую точность масок Собела в отношении ошибок 2-го рода при СКО $\sigma>60$ (рис. 4в).

Часто для сглаживания помех на изображении перед этапом выделения контура применяют раз- личные процедуры фильтрации, например, предложенную в [10]. С использованием предлагаемой программной модели рассмотрим, насколько использование простого усредняющего фильтра позволяет снизить ошибки 1-го и 2-го рода при выделении контуров зашумленных изображений.

На рисунке 5 показан результат выделения контуров из зашумленного изображения (рис. 1а) с СКО $\sigma=20$ после применения предварительного усредняющего фильтра с маской $3 \times 3$ элемента аналогичными тремя процедурами: на основе масок Превитта (5), Собела (6) и лапласиана гауссиана (8).

Сопоставляя рисунки 3 и 5, можно увидеть, что операция предварительного сглаживания зашумленного изображения позволяет получить более качественное выделение контуров. На рисунке 6 показаны аналогичные рисунку 4 зависимости частот ошибок 1-го и 2-го рода от СКО для трех указанных

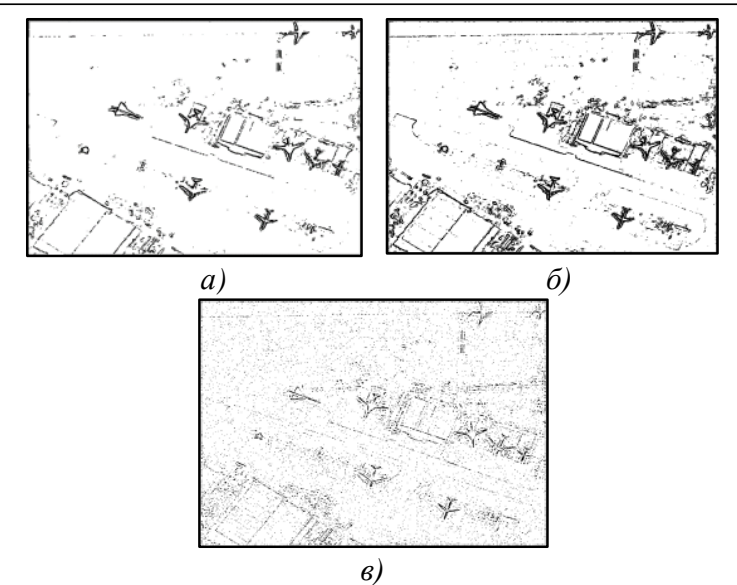

Рис. 5. Получение контура из предварительно усредненного зашумленного изображения: а) маски Превитта (5), б) маски Собела (6), в) лапласиан гауссиана (8)

Fig. 5. Obtaining a contour from a previously averaged noisy image: a) Prewitt masks (5), б) Sobel masks (6), в) Laplacian of Gaussian (8) 


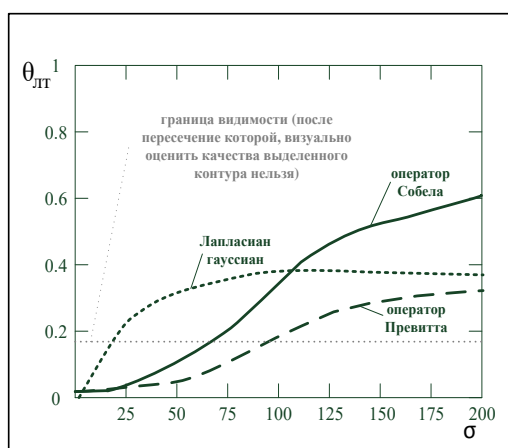

a)

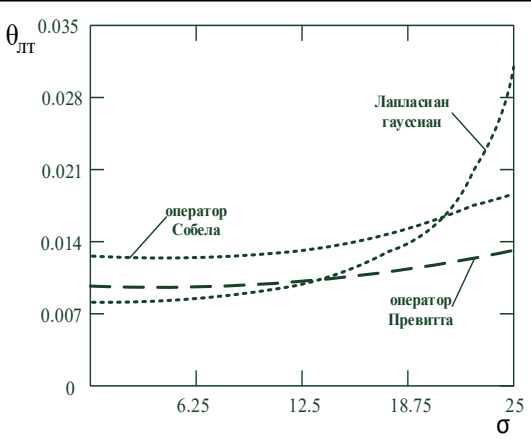

б)

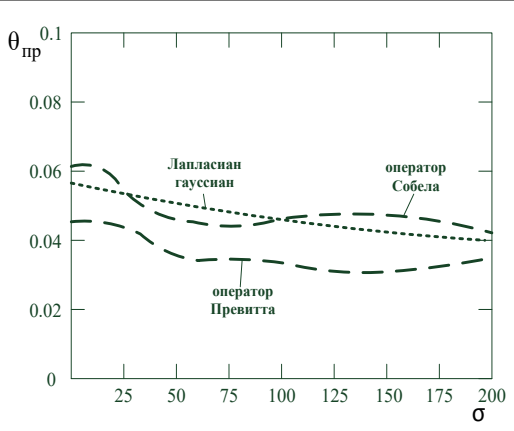

в)

Рис. 6. Ошибки операторов выделения контура предварительно сглаженного изображения: а) ошибки 1-го рода (во всем диапазоне б), б) ошибки 1-го рода (при $\sigma<25)$,

в) ошибки 2-го рода (во всем диапазоне $\sigma$ )

Fig. 6. Errors of contour extracting operators of a previously smoothed image: a) errors of the 1st kind (in full range of $\sigma$ ), б) errors of the 1st kind (when $\sigma<25$ ), в) errors of the 2 nd kind (in full range of $\sigma$ )

процедур выделения контура из сглаженного зашумленного изображения.

Сравнивая рисунки 4 и 6, можно оценить снижение частоты ошибок для каждой из исследуемых процедур выделения контура при использовании предварительной процедуры усреднения изображения.

На рисунке 7 рассмотренная программная модель исследования эффективности процедур выделения контуров изображений представлена в схематическом виде, где блоками показаны реализуемые вычислительные операции (2)-(11).

Таким образом, предлагаемая программная модель позволяет наглядно исследовать эффективность различных процедур выделения контуров изображений во всем возможном диапазоне наличия аддитивных гауссовских помех.

\section{Заключение}

Так как АГШ является наиболее распространенным и часто встречающимся на практике, предлагаемая программная модель может быть использована для исследования эффективности процедур выделения контуров изображений, полученных в неблагоприятных условиях, таких как туман, повышенная влажность, предельно низкая освещенность, критические температуры, вибрации фотоприемника.

Разработанная программная модель исследования эффектив- ности выделения контуров в условиях АГШ реализована в виде самостоятельной программы [8], однако может быть добавлена в качестве дополнительного компонента в программные модули существующих пакетов программ по обработке изображений.

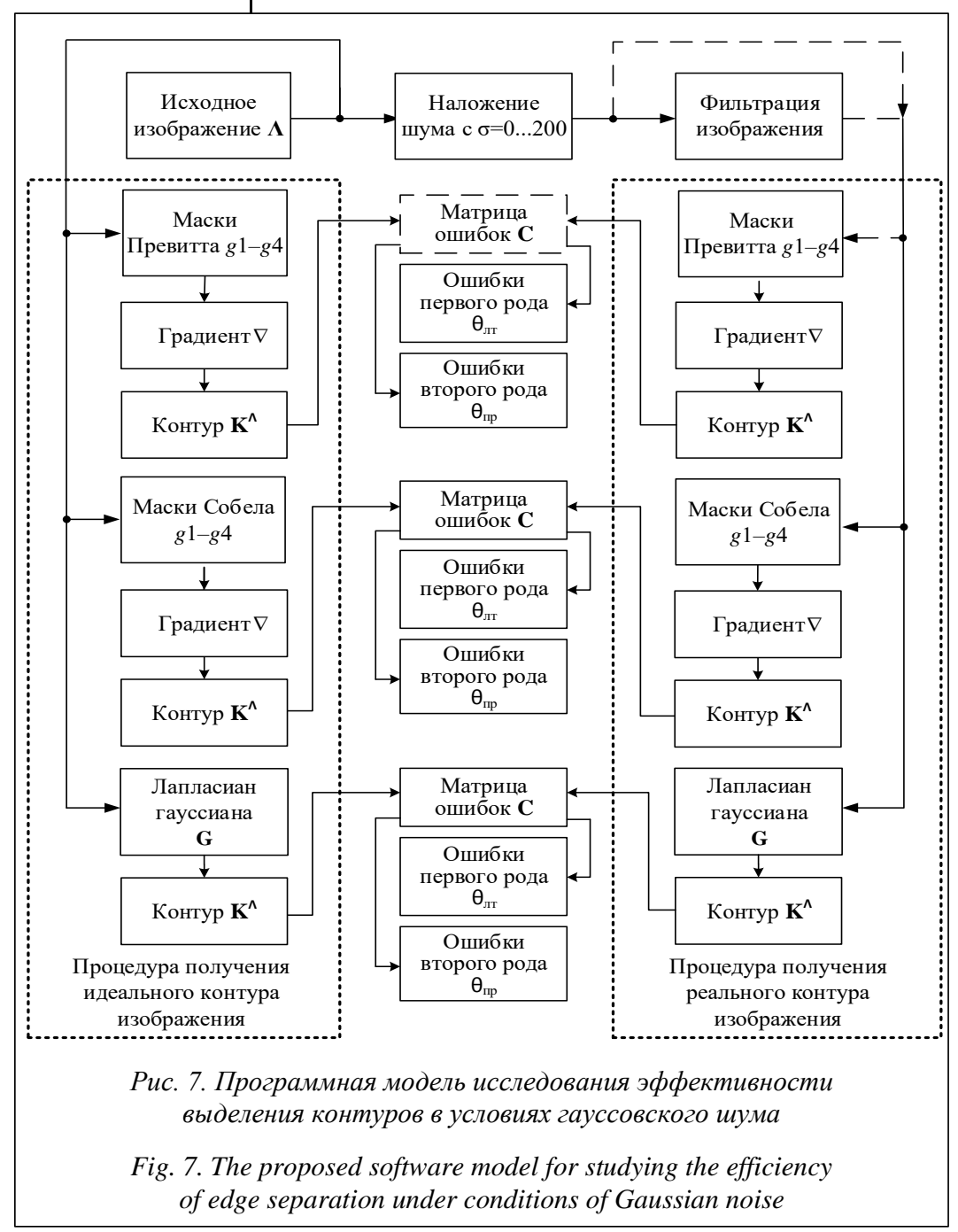




\section{Лuтература}

1. Гонсалес Р., Вудс Р. Цифровая обработка изображений. М.: Техносфера, 2012. 1104 с.

2. Фурман Я.А., Кревецкий А.В., Передреев А.К., Роженцов А.А., Хафизов Р.Г., Егошина И.Л., Леухин А.Н. Введение в контурный анализ; приложения к обработке изображений и сигналов. М.: Физматлит, 2003. 592 с.

3. Местецкий Л.М. Непрерывная морфология бинарных изображений: фигуры, скелеты, циркуляры. М.: Физматлит, 2009. 288 c.

4. Сальников И.И. Растровые пространственно-временные сигналы в системах анализа изображений. М.: Физматлит, 2009. 248 c.

5. Сирота А.А., Соломатин А.И. Обнаружение и оценка параметров перепада яркости в задаче контурного анализа объектов // Автометрия. 2009. № 5. С. 59-69.

6. Котов В.М., Шкердин Г.Н., Шкердин Д.Г., Котов Е.В.
Оконтуривание изображений посредством брэгговской акустооптической дифракции в два порядка // Радиотехника и электроника. 2011. Т. 56. № 1. С. 63-66.

7. Нагалин А.В., Самойлин Е.А., Пантюхин М.А., Беляев Р.В. Способ пространственной адаптации градиентных масок для оконтуривания объектов на зашумленных изображениях // Радиотехника. 2016. № 8. С. 26-31.

8. Карпов С.А. Программа оценки эффективности выделения контуров цифровых изображений на фоне аддитивного гауссовского шума. Свид. 2018613303 об официальной регистрации программы для ЭВМ, Рос. Федерация, № 2018610319/69; заявл. 16.01.2018; опубл. 07.03.2018.

9. Петров А.И. Статистическая теория радиотехнических систем. М.: Радиотехника, 2003. 400 с.

10. Самойлин Е.А., Шипко В.В. Программно-реализуемый метод межканальной градиентной реконструкции цветных цифровых изображений // Программные продукты и системы. 2014. № 2. C. $157-160$.

\title{
A software model for studying the effectiveness of procedures for extracting contours of noisy images
}

\author{
E.A. Samoylin ${ }^{1}$, Dr.Sc (Engineering), Professor, es977@mail.ru
}

S.A. Karpov ${ }^{1}$, Adjunct, sergei-andreevich@bk.ru

${ }^{1}$ Military Scholastic-Scientific Centre of the Air Forces "Zhukovsky and Gagarin Air Forces Academy", Voronezsh, 394064, Russian Federation

Abstract. The article develops one of the directions of digital image processing - extracting their contours. It is necessary to solve this task in order to recognize objects in the image (by geometry, the angles between contour fragments, etc.), to improve image visual quality, to emphasize the boundaries of objects of interest, contrasting, etc.

Typically, digital images are recorded in difficult and adverse conditions, which leads to noise generation described by an additive Gaussian noise model. Therefore, the authors propose a software model that allows investigating the effectiveness of various methods, algorithms and procedures to extract noisy image contours.

The model is based on comparing an image contour from a noisy image to an ideal contour of a similar image without noise. Then it is supposed to calculate the errors of detection of contour elements of the first (false alarm) and the second (pass) kind. In this case, the error calculation is carried out over the entire possible range of the mean square deviation $\sigma$ of additive Gaussian noise.

The paper gives some examples of extrating contours of real scene noisy images, as well as dependences of error frequencies of the first and second kinds on the mean square deviation of the amplitude noise from $\sigma=0$ to $\sigma=200$ (images at 256 levels of brightness amplitude quantization) for three procedures for contour extraction: based on Prewitt masks, Sobel mascs and the Laplacian of Gaussian. In addition, the authors study the influence of a noisy image linear filtering procedure on the decrease in the frequency of errors in the extrating contours of the first and second kinds.

Keywords: image, outlines, additive Gaussian noise, gradient masks, Prewitt masks, Sobel masks, Laplacian of Gaussian.

1. Gonsales R.C., Woods R.E. Digital Image Processing. Pearson Education, Prentice Hall Publ., 2012, 1104 p.

2. Furman Ya.A., Krevetsky A.V., Peredreev A.K., Rozhentsov A.A., Khafizov R.G., Egoshina I.L., Leukhin A.N. Introduction to Contour Analysis; Applications for Image and Signal Processing. Ya.A. Furman (Ed.). 2nd ed. Moscow, Fizmatlit Publ., 2003, 592 p.

3. Mestetsky L.M. Continuous Morphology of Binary Images: Figures, Skeletons, Circulars. Moscow, Fizmatlit Publ., 2009,288 p.

4. Salnikov I.I. Raster Spaciotemporal Signals in Image Analysis Systems. Moscow, Fizmatlit Publ., 2009, 248 p.

5. Sirota A.A., Solomatin A.I. Detection and estimation of brightness difference parameters in object boundary analysis. Optoelectronics, Instrumentation and Data Processing. 2009, no. 5, pp. 59-69 (in Russ.).

6. Kotov V.M., Shkerdin G.N., Shkerdin D.G., Kotov E.V. Contouring of images by means of the bragg acoustooptical diffraction into two orders. J. of Communications Technology and Electronics. 2011, vol. 56, no. 1, pp. 63-66.

7. Nagalin A.V., Samoylin E.A., Pantyukhin M.A., Belyaev R.V. Spatial adaption method of gradient masks to segment countours of objects on the noised images. J. Radioengineering. 2016, no. 8, pp. $26-31$ (in Russ.)

8. Karpov S.A. The program for assessing the effectiveness of extracting contours of digital images in presence of additive Gaussian noise. Certif. of registration of a computer program 2018613303. No. 2018610319/69, 1 p. (in Russ.).

9. Petrov A.I. The Statistical Theory of Radio Systems. Moscow, Radiotekhnika Publ., 2003, 400 p.

10. Samoylin E.A., Shipko V.V. A software-implemented interchannel gradient reconstruction method for color digital images. Software \& Systems. 2014, no. 2, pp. 157-160 (in Russ.). 